\title{
Sigatoka negra (Mycosphaerella fijiensis Morelet) y seguridad alimentaria. Escenarios biocli- máticos en bananos bajo efecto del cambio climático en Ciego de Ávila, Cuba \\ Black Sigatoka (Mycosphaerella fijiensis Morelet) and food security.

\author{
Bio-climate Scenarios in bananas under the effect of climate change in Ciego de Avila, Cuba
}

Hernández-Mansilla Alexis Augusto ${ }^{1 *}$, Sorí-Gómez Rogert ${ }^{2}$, Valentín-Pérez Yadira ${ }^{1}$; López-Mayea Aliana ${ }^{1}$, Córdova-García Orlando ${ }^{1}$, Benedico-Rodríguez Oscar ${ }^{1}$

\begin{tabular}{l}
\hline DatoS del Articulo \\
\hline${ }^{\text {I} C e n t r o ~ M e t e o r o l o ́ g i c o ~ P r o v i n c i a l ~ C i e g o ~}$ \\
de Ávila - Órgano de base de la Asocia- \\
ción Cubana de Técnicos Agrícolas y \\
Forestales (ACTAF). Filial Ciego de \\
Ávila. Marcial Gómez 401, esq. Estrada. \\
Ciego de Ávila, Cuba. CP: 65200. \\
2EphysLab, Departamento de Física \\
Aplicada, Facultad de Ciencias de \\
Ourense, Universidad de Vigo, Ourense, \\
España. CP: 32004. \\
*Dirección de contacto: \\
Hernández-Mansilla Alexis Augusto \\
Centro Meteorológico Provincial Ciego de \\
Ávila - Órgano de base de la Asociación \\
Cubana de Técnicos Agrícolas y Foresta- \\
les (ACTAF). Filial Ciego de Ávila. \\
Marcial Gómez 401, esq. Estrada. Ciego \\
de Ávila, Cuba. CP: 65200. \\
E-mail: alexis.hernandez@cav.insmet.cu \\
ahmansilla@gmail.com
\end{tabular}

\section{Palabras clave:}

Cambio climático,

Sigatoka negra,

Mycosphaerella fijiensis,

enfermedades de bananos,

seguridad alimentaria.

J Selva Andina Biosph. 2016; 4(2):59-70.

\section{Historial del artículo.}

Recibido abril, 2016.

Devuelto septiembre 2016

Aceptado septiembre, 2016

Disponible en línea, noviembre 2016.

\begin{tabular}{l}
\multicolumn{1}{c}{$\begin{array}{c}\text { Editado por: } \\
\text { Selva Andina } \\
\text { Research Society }\end{array}$} \\
\hline Key words: \\
\hline \\
Climate change, \\
Sigatoka negra, \\
Mycosphaerella fijiensis, \\
banana diseases, \\
food security.
\end{tabular}

\section{Resumen}

Evidencias negativas del Cambio Climático, de elevada trascendencia para el futuro productivo de la esfera agrícola y la necesidad de una soberanía y seguridad alimentaria, imponen desarrollar investigaciones para conocer el comportamiento fenológico de especies vegetales y sus plagas; fundamentalmente las de relevancia económica. El objetivo del trabajo consiste en interpretar el comportamiento epidemiológico de la enfermedad Sigatoka negra (Mycosphaerella fijiensis Morelet) en bananos para los años 2020 y 2025 bajo efectos del cambio climático en Ciego de Ávila. Se confeccionaron escenarios bioclimáticos utilizando datos diarios de temperatura máxima, temperatura mínima y el acumulado de precipitación de 14 días generados por el Modelo Climático Regional "PRECIS CARIBE" con condiciones de frontera del modelo global ECHAM-4 para proyecciones climáticas futuras bajo escenario de emisiones A2 y B2. Se obtuvieron indicadores fundamentales de valoración de esta patología: la "Suma de Velocidades" y el "Estado de Evolución" de 4 hojas. Los resultados muestran Sumas de Velocidades por encima de 11000 unidades termo-fisiológicas, así como un continuo y progresivo incremento del "Estado de Evolución" con valores superiores a 500 unidades durante todos los años analizados. Se infiere se produzca un incremento en el número de tratamientos y el costo fitosanitario, lo que supone la necesidad de trazar medidas de manejo integrado que incluyan principios agroecológicos; así como la búsqueda de variedades resistentes para sustitución de las susceptibles a la enfermedad que se emplean en la actualidad y consolidar la adaptación al cambio climático y la resiliencia en este agroecosistema.

() 2016. Journal of the Selva Andina Biosph. Bolivia. Todos los derechos reservados.

\section{Abstract}

The negative evidence of climate change of high importance for the future agricultural production and the need of food sovereignty and security, impose to develop research to predict the phenology of plants and their pests, mainly those of economic importance. The aim of this work is to interpret the epidemiological behavior of the black Sigatoka disease (Mycosphaerella fijiensis Morelet) in banana for the years 2020 and 2025 under the effects of climate change in Ciego de Avila. There were made bioclimatic scenarios using daily data of maximum temperature, minimum temperature and accumulated rainfall of 14 days generated by the Regional Climatic Model "PRECIS Caribbean" with boundary conditions of the global model ECHAM - 4 for future climate projections under scenarios of emission A2 and B2. The key indicators of this disease: "Sum of Speed " and the "Evolution state" of 4 leaves were obtained. The results show Sums speeds above 11000 thermo-physiological units, as well as a continuous and progressive increase in the "Evolution State" with values above 500 units during the analyzed years. We infers will occur an increase in the number of treatments and the plant protection cost, which implies the need to draw integrated management measures that include agro-ecological principles, search for resistant varieties to replace those susceptible to the disease that are currently used and strengthen the climate change adaptation and the resilience in the agricultural ecosystem. 


\section{Introducción}

La disponibilidad de alimentos para todos "seguridad alimentaria", es sin dudas uno de los elementos de mayor preocupación de la sociedad actual, bajo un estatus de crisis económica, energética y climática que impone la búsqueda de garantías alimentarias para estimados con un creciente aumento de un $70 \%$ en comparación con los actuales niveles productivos y en correspondencia con el crecimiento demográfico que se espera para la década del 2050 (De Schutter 2010). Ello se une a previsiones de disminución de un 3 a $16 \%$ de la capacidad productiva agrícola para el año 2080 (Cline 2007) como consecuencias de los efectos del cambio climático, de ocurrencia inequívoca (IPCC 2007). El mismo, se materializa en una mayor frecuencia de fenómenos meteorológicos extremos como sequías e inundaciones, así como disminución de las precipitaciones predecibles, con secuelas graves para la capacidad de alimentarse en determinadas regiones y comunidades, que implican la desestabilización de los mercados y reducen la seguridad del suministro de los alimentos básicos (Warrington 2008, De Schutter 2010).

Es importante, tomar en consideración los efectos del cambio climático sobre los cultivos. Según criterios de Rosenzweig et al. (2007), muchos de los sistemas naturales y manejados muestran un número significativo de cambios, que incluyen impactos en los ciclos estacionales y de vida (fenológicos) de las especies vegetales, además de los cambios en las manifestaciones en variables climáticas claves asociadas con el crecimiento de los cultivos, como la precipitación, temperatura y otras que pueden afectar severamente la producción agrícola (Cline 2007). Súmense, aquellas asociadas con el desarrollo de plagas, organismos decisivos para el rendi miento de los cultivos y sobre los que recaen pérdidas entre un $20 \%$ y un $40 \%$ de la cosecha potencial de alimentos en países en desarrollo (PNUMA 2009, citado por De Schutter 2010). Se plantea que por efecto del cambio climático los insectos plagas serán más abundantes en la medida que la temperatura aumenta, así como se observarán modificaciones en las etapas y en las tasas de desarrollo de ciertos patógenos y alteraciones de la resistencia de los hospedantes, que darán lugar a cambios en la fisiología de las interacciones entre hospedante y el patógeno. Producto de las variaciones en los valores de temperatura y humedad, se puede predecir que muchos patógenos incrementarán su severidad, situación que conduce a aumentar el uso de pesticidas químicos, costos de producción y problemas ambientales (Altieri \& Nicholls 2008).

En Cuba, el cultivo del banano es un producto agrícola básico para la alimentación de sus pobladores. Normalmente se consume como fruta y en variadas formas de cocción, por lo que ocupa un lugar decisivo en la seguridad alimentaria local al integrar su dieta diaria. A su vez es un importante reglón dentro de la economía, tanto por sus aportes en divisas por ventas de fruta fresca al turismo, como por suplir las demandas crecientes de la población, situación que según los antecedentes planteados sobre las posibles afectaciones del cambio climático pone en riesgo la productividad de este cultivo, por demás extendido en la mayoría de las zonas agrícolas de Cuba. La producción en el país durante los años 2011, 2012 y 2013 alcanzó valores de 250 000, 195 496 y 150336 toneladas respectivamente (FAOSTAT 2015).

Actualmente y en particular los compromisos productivos de Ciego de Ávila destinados a satisfacer gran parte de las necesidades del balance nacional, 
tienden a mantener un área de 1570 ha de banano y 3174 ha de plátanos, distribuidas fundamentalmente en la Empresa de Cultivos Varios "La Cuba", Cooperativas de Producción Agropecuarias y Cooperativas de Créditos y Servicios del municipio Baraguá, una de las localidades de mayor importancia en la nación dedicadas a este cultivo (Dirección de Cultivos Varios. Delegación Territorial de la Agricultura en Ciego de Ávila 2011). Las extensiones dedicadas a la siembra de este cultivo en la provincia evidencian el papel de estas producciones en la alimentación y la economía de esta provincia y país.

En un sistema de explotación bananero, los rendimientos y los costos de producción dependen no solo de una esmerada agrotécnia, sino también del resultado de la incidencia y efectivo control de plagas. Dentro de estas y para este cultivo, la Sigatoka negra causada por Mycosphaerella fijiensis Morelet, se reconoce como la enfermedad de mayor importancia en el mundo (Stover \& Simmonds 1987) y su presencia en Cuba de acuerdo a informaciones de Vidal (1992) data de finales de 1990 en Clones Cavendish gigante, Parecido al Rey, Gran enano y Cavendish enano (Musa spp., Subgrupo Cavendish, AAA), Macho 3/4, CEMSA 3/4, todas susceptibles a este fitopatógeno. Esta enfermedad de no mantener un eficiente control provoca daños y pérdidas significativas en el rendimiento, según Stover \& Simmons (1987) el $27 \%$ del costo total de una caja de bananos en Centroamérica se destina a la protección de esta enfermedad. Otros autores como Romero (1993) en Costa Rica señalaron que hasta un 5\% del total de la producción puede perderse a pesar de un número considerable de tratamientos para su control. Sus daños en el cultivo son a causa de una disminución de la actividad fotosintética y una maduración previa de los frutos como consecuencia de la 61 necrosis foliar, la muerte de sus hojas. Esto impone regímenes con un número elevado de tratamientos con fungicidas sistémicos, los cuales requieren de avanzada tecnología, de la señalización bioclimática y de un continuo monitoreo para evitar el peligro de la aparición de la fungo resistencia, que de estar presente solo conduce a la pérdida de la efectividad del control de la enfermedad, a la inutilización de los ingredientes activos y a la necesidad de recurrir a la introducción de nuevas moléculas que solo implicarán mayores expendios. Es un hecho que la situación fitosanitaria es compleja, exige de la utilización de sistemas de manejo integrado de alta eficiencia para poder garantizar elevados rendimientos y aumentar la rentabilidad, por lo cual se debe reflexionar sobre ¿Cómo enfrentar los posibles impactos del cambio climático en este cultivo y fundamentalmente sus efectos sobre el desarrollo epidemiológico de esta enfermedad? Ello constituye un problema que requiere de la construcción de escenarios bioclimáticos que expresen el posible comportamiento epidemiológico de Sigatoka negra, para lo cual, los Modelos Climáticos constituyen herramientas imprescindibles, varios utilizados y evaluados por el Panel Intergubernamental de Cambio Climático (IPCC) de la Organización de Naciones Unidas.

Carter et al. (1994), establecen que los escenarios son "una descripción coherente, internamente consistente y plausible de un posible estado futuro del mundo". Las principales fuentes de incertidumbre en los escenarios de cambio climático (utilizados para evaluar los impactos potenciales) son: a) incertidumbre en las emisiones; b) incertidumbres en la variabilidad natural; c) incertidumbres asociadas a los modelos climáticos.

En la generación de Escenarios de Cambio climático y su aplicación a estudios de impacto se hace 
necesario considerar más de un escenario socioeconómico-ambiental. Este tipo de escenarios son documentados por el IPCC en el Reporte Especial de Escenarios de Emisiones (SRES, por sus siglas en inglés), y aplicados con diferentes Modelos de Circulación General (MCG), (Nakičenovič \& Swart 2000).

Con el objetivo de reducir las predicciones a escala regional y local se han desarrollado Modelos Climáticos Regionales (MCR) como el PRECIS CARIBE implementado en Cuba, o manejando técnicas estadísticas de reducciones de escala para lograr una mejor representación de parámetros climáticos a escala local. A su vez pueden ser empleados junto a parámetros biológicos y eco fisiológicos de organismos vivos mediante la elaboración escenarios bioclimáticos, información que permite predecir el comportamiento futuro de estos ante nuevas condiciones climáticas. Ello posibilita trazar las medidas adecuadas para la adaptación dentro de programas de acciones de planificación económica y con ello contribuir a garantizar las producciones de los cultivos agrícolas, la seguridad alimentaria y el enfrentamiento al cambio climático. De ahí que el objetivo de este trabajo consista en interpretar el comportamiento epidemiológico de la enfermedad Sigatoka negra (M. fijiensis) en bananos para los años 2020 y 2025 en Ciego de Ávila bajo efectos del cambio climático mediante la confección de escenarios bioclimáticos.

\section{Materiales y métodos}

El estudio se desarrolló dentro de las actividades investigativas del Proyecto Nacional: "Cambio Climático. Elaboración de escenarios para el desarrollo fenológico, situación fitosanitaria de cultivos agrícolas y zonas de interés medio ambiental en
Ciego de Ávila” que ejecuta el Centro Meteorológico Provincial de Ciego de Ávila y que pertenece al Programa Nacional de Ciencia y Tecnología: Cambio Climático en Cuba: Impactos, mitigación y adaptación. Para la confección de los escenarios bioclimáticos de Sigatoka negra en bananos, se emplearon salidas diarias de variables meteorológicas del Modelo Climático Regional (MCR) PRECIS CARIBE, que a su vez utilizó datos de frontera generados por el Modelo Global Echam-4 (Taylor et al. 2013) utilizando escenario de emisiones de Gases de Efecto Invernadero SRES A2 (mayor concentración de $\mathrm{CO}_{2}$ ) y $\mathrm{B} 2$ (menor concentración de $\mathrm{CO}_{2}$ ). Se seleccionaron los años 2020 y 2025 para predecir la aparición y desarrollo de estas enfermedades a plazos operativos, cortos. Para la identificación de periodos de afectación sería posible proponer planes de adaptación fundamentados en la ejecución de medidas efectivas de manejo fitosanitario, que conduzcan a la resiliencia en especial para Ciego de Ávila, evitar daños al cultivo y afectación en los índices de rendimiento, imprescindibles en el futuro para esta agroindustria.

Los datos de las variables meteorológicas utilizadas, temperatura máxima (Tx), temperatura mínima (Tn) y precipitación se corresponden a las coordenadas geográficas $22^{\circ} \mathrm{N}$ y $78.5^{\circ} \mathrm{W}$, representativas para el macizo productivo de bananos Cavendish (AAA) sembrados sobre suelo ferralítico rojo, ubicado en la Empresa Cultivos Varios "La Cuba" y Cooperativas de Producción Agropecuaria, pertenecientes al municipio Baraguá en la provincia de Ciego de Ávila. Para el uso de los datos modelados se tuvo en cuenta la representación de la estacionalidad mediante criterios de Lecha et al (1994), además de la ocurrencia y magnitud de valores extremos. En el caso específico de la precipitación extremadamente compleja de predecir, se corrigieron los datos modelados mediante la obtención de un coeficiente de 
corrección bimestral obtenido según las diferencias con datos diarios de precipitación en el período 1992 - 2009, registrada en la estación pluviométrica de " $L a C u b a$ " representativa del área de estudio y perteneciente al Instituto Nacional de Recursos Hidráulicos.

El comportamiento epidemiológico de Sigatoka Negra se obtuvo sobre la base de la determinación del Estado de Evolución (EE) y la proyección de la fluctuación de las Sumas de Velocidades (SV) o sumas térmicas, que permiten considerar los índices favorables para el desarrollo de esta enfermedad. Para su discusión se confeccionaron escenarios bioclimáticos que expresan el desarrollo de la enfermedad durante los años escogidos. Las SV de 7 días (semanales) se determinaron mediante la ecuación recomendada por Porras-González \& Pérez-Vicente (1997) fundamentada en el método termofisiológico de Livingstone para el desarrollo de la enfermedad (I), y el EE de la enfermedad mediante la ecuación predictiva (II) propuesta por Pérez-Vicente et al. (2000).

\section{SV= 7.8*Tmáx. + 79.16*Tmín (I)}

EE 4H = (8.09)*acumulado de 14 días de LL (mm) (II)

\section{Resultados}

El escenario bioclimático para el 2020 bajo condicionantes A2 (Figura 1), muestra durante los primeros meses de enero a mayo, índices de EE que varían entre 500 y 600 unidades, que seguidamente ascienden a niveles estables entre 900 y 1000 unidades aproximadamente durante el período de junio a septiembre. Posteriormente se observa un aumento brusco en el mes de octubre que sobrepasa las 3000 unidades, para luego volver a descender en los meses de noviembre y diciembre a valores entre 500 y 900 unidades. Los valores de las sumas térmicas que se predicen señalan índices superiores a 11000 unidades durante todo el año que posibilitan condiciones favorables para el desarrollo de la enfermedad.

En el escenario bioclimático B2 se observa mayor variabilidad del índice EE con respecto al observado en el A2 así como la disminución de valores máximos durante el año. Desde enero hasta mayo los valores de EE se predice oscilen entre 400 y $700 \mathrm{u}$, para luego aumentar desde finales de junio hasta alcanzar valores máximos en julio $(\sim 1600 \mathrm{u})$, finales de octubre $(\sim 1800 \mathrm{u})$ y diciembre $(\sim 1700 \mathrm{u})$.

Para ambos escenarios las condiciones térmicas justifican se esperen valores de sumas térmicas superiores a las $11000 \mathrm{u}$; condiciones favorables que garantizan el proceso infeccioso y el desarrollo de la enfermedad. En ambos escenarios se representa correctamente el ciclo estacional de las temperaturas en la provincia, con máximos durante el verano boreal y mínimos durante diciembre, enero y febrero. Sin embargo, en el escenario B2 la variabilidad tanto en valores de temperatura máxima (Tx) como mínima (Tn) es mayor. Ello es además observado en los valores de precipitación acumulada en 14 días. El escenario bioclimático para el 2025 (Figura 2), señala para condiciones $\mathrm{A} 2$ que los valores de EE de la enfermedad oscilarán entre 500 y 2500 u. Su dinámica expresa tres momentos con diferentes niveles de enfermedad durante el año, uno para los primeros meses enero, febrero, marzo y abril entre 500 y $600 \mathrm{u}$, un segundo de mayo a septiembre cuando el EE oscila entre 500 y 1400 u y un tercero entre octubre y diciembre con índices entre 600 y 1993 unidades. 
Figura 1 Comportamiento epidemiológico de Sigatoka negra (Mycosphaerella fijiensis Morelet) bajo efecto del cambio climático en bananos.

Escenarios bioclimáticos para el año 2020 bajo escenarios de emisiones A2 (izquierda) y B2 (derecha)

Escenarios A2
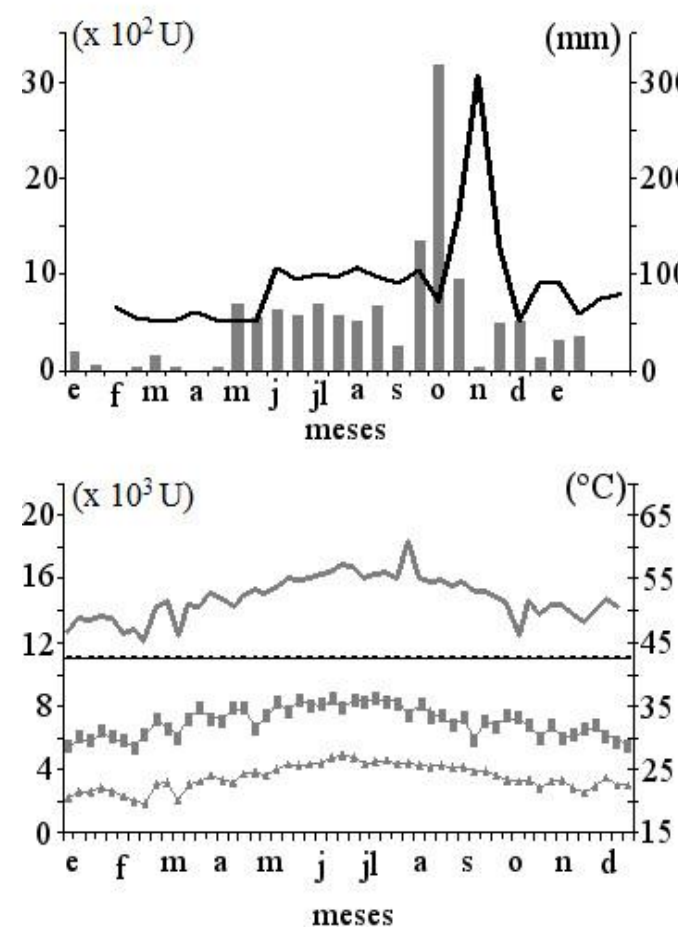

Respecto a los valores que alcanzan las temperaturas, las mismas se muestran favorables para que el índice SV supere las 11000 u durante todo el año. En comparación, el comportamiento de la enfermedad en la condición B2 para este mismo año (Figura 2), muestra de enero a junio índices de EE superiores a $450 \mathrm{u}$ e inferiores a $722 \mathrm{u}$, salvo un incremento

\section{Discusión}

Este ascomycete ( $M$. fijiensis) constituye una enfermedad endémica con recurrencia durante todo el año y evolución y desarrollo biológico de alta dependencia a la temperatura y la humedad. Ello condiciona que su comportamiento epidemiológico generalmente se ajuste a los dos períodos climatológicos bien definidos que posee el país, uno poco lluvioso (noviembre a abril) acompañado de bajas
Escenarios B2
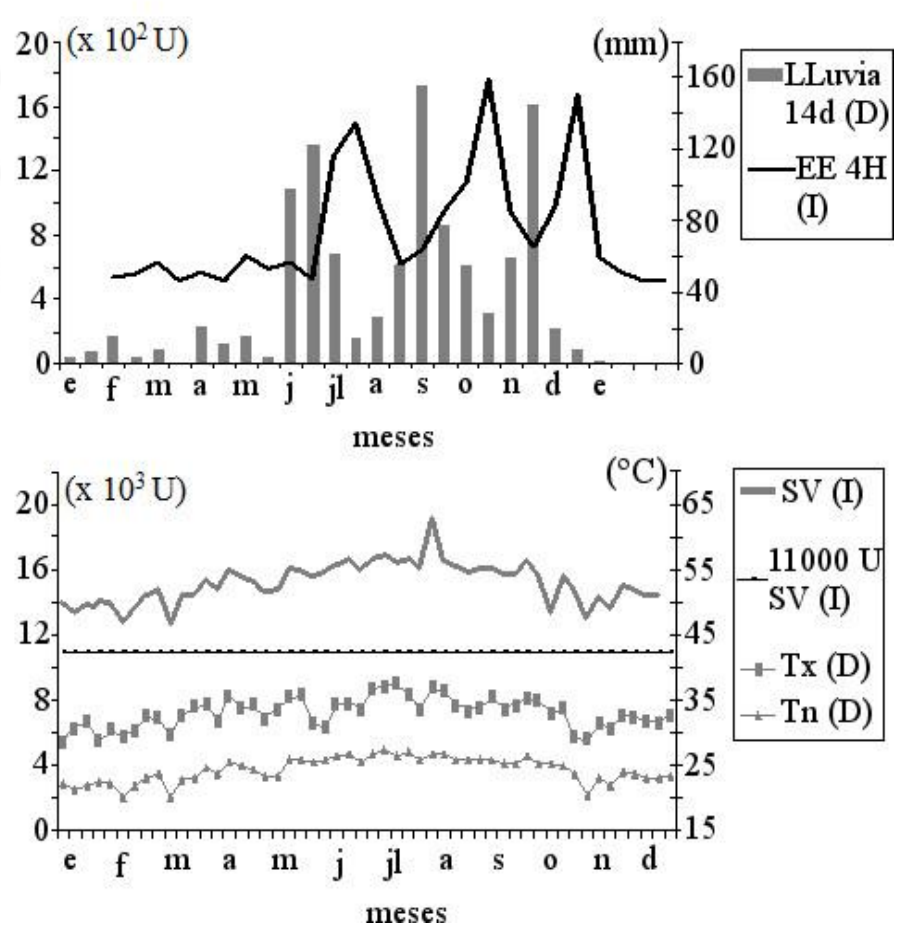

a finales del mes de junio y en los primeros días de julio, momento a partir del cual se estabiliza nuevamente con una fluctuación entre 500 y 900 u hasta noviembre. Para diciembre se predice un ascenso del EE hasta las $1850 \mathrm{u}$. Las sumas de velocidades en ambos escenarios se mantienen superiores a las 11000 unidades para todo el año.

temperaturas donde la enfermedad evoluciona más lentamente y otro lluvioso (mayo a octubre) con temperaturas cálidas (oscilan generalmente con valores medios entre 24 y $28^{\circ} \mathrm{C}$, índices de esta variable qué según Stover (1980) en Honduras, y Fouré (1982) en Camerún, permiten una aparición entre 15 y 17 días de los síntomas iniciales después de la infección) y en presencia de alta humedad relativa, que condicionan una rápida evolución, un acortamiento del ciclo y un mayor número de indi- 
viduos enfermos. Al respecto, Pérez-Vicente et al. (2000) plantea que en la época más seca y fría en
Cuba el período de incubación máximo es de 25 días y de 15 a 17 días en la más caliente y húmeda.

Figura 2 Comportamiento epidemiológico de Sigatoka negra (Mycosphaerella fijiensis Morelet) bajo efecto del cambio climático en bananos.

Escenarios bioclimáticos para el año 2025 bajo escenarios de emisiones A2 (izquierda) y B2 (derecha)

Escenarios A2
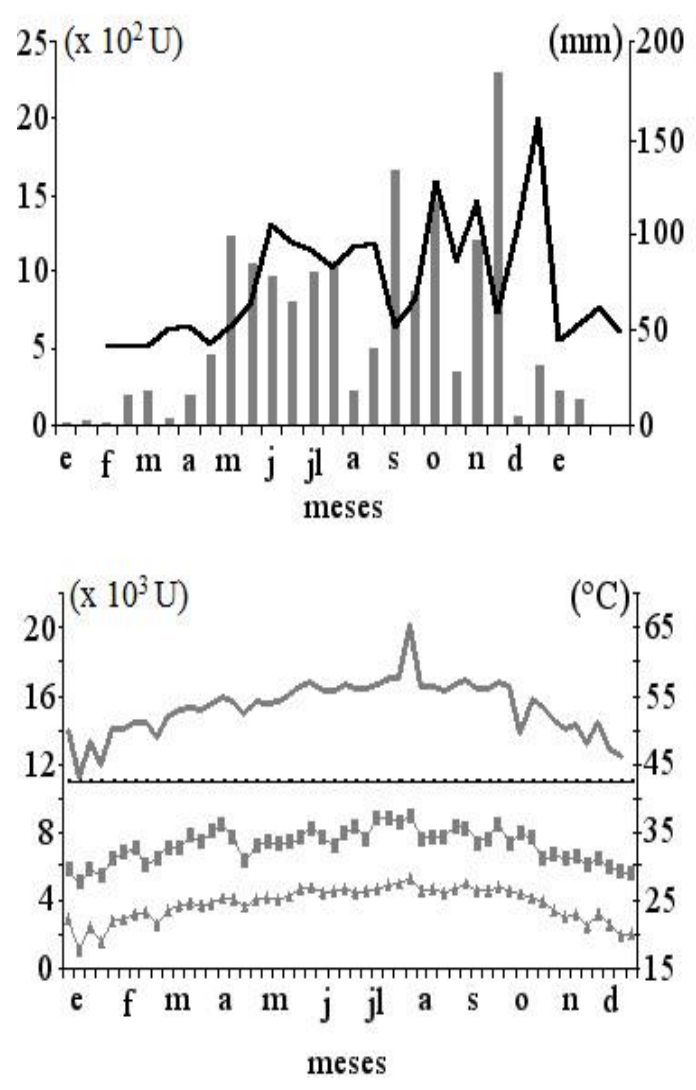

Esta situación explica la manifestación de un comportamiento epidemiológico de referencia de acuerdo a condiciones de temperatura y humedad (con fuerte dependencia del régimen pluviométrico) para su desarrollo. Sin embargo, debido al cambio climático su epidemiología puede cambiar, lo cual se demuestra en los escenarios bioclimáticos para el 2020 y 2025 (Figuras 1 y 2); estos señalan un desarrollo evolutivo intenso y de elevados índices de Sigatoka negra en bananos tanto para condiciones 65

\section{Escenarios B2}
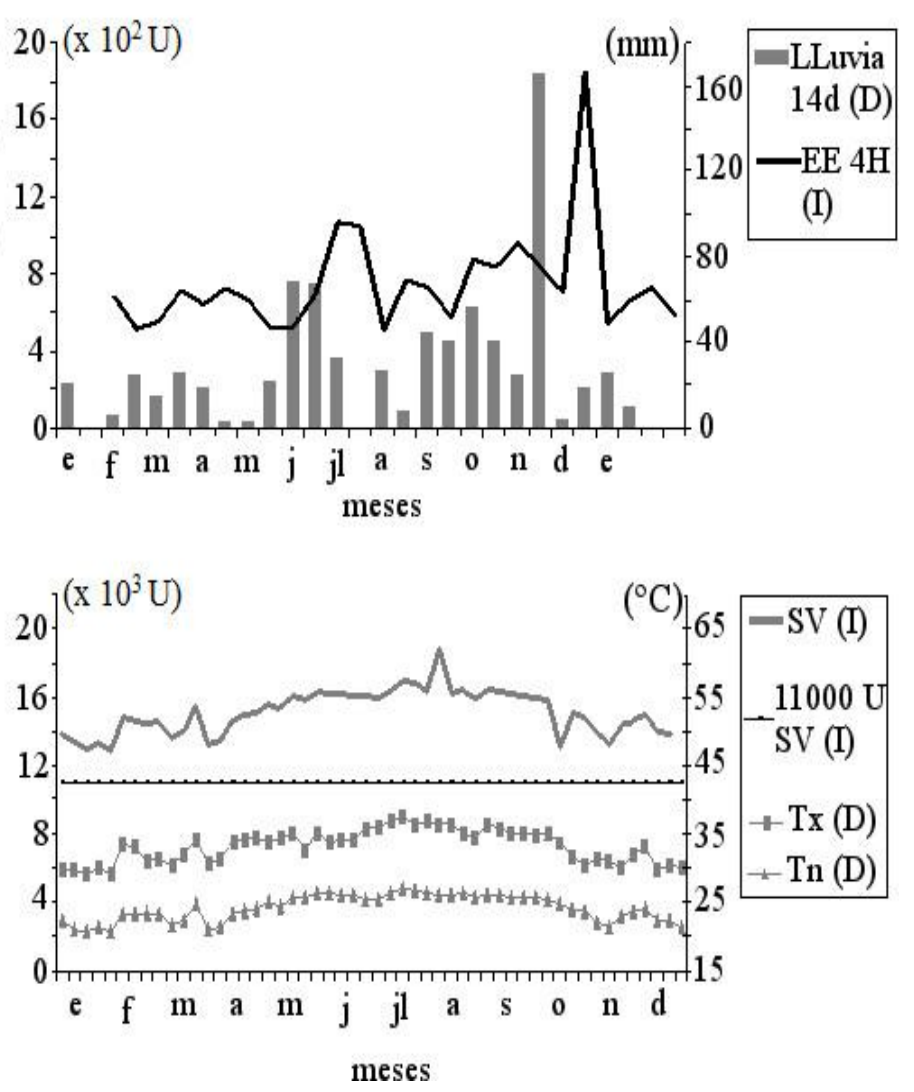

de emisiones A2 como B2, a causa de los efectos de temperaturas favorables predominantes durante todos los meses del año. Ello además está asociado con la precipitación, particularmente durante el período lluvioso correspondiente a los meses de mayo a octubre, aunque se predice una incidencia regular de esta variable con niveles significativos hasta el mes de noviembre. Aun cuando existe incertidumbre en las predicciones a mediano y largo plazo, de las mismas se puede interpretar que el 
efecto del cambio climático provocará un aumento de los índices epidemiológicos de la enfermedad y en correspondencia se elevará el número de tratamientos de fungicidas, los cuales se requerirán en una frecuencia continua y sistematizada acorde a los índices que se establecen para orientar los tratamientos y que exige el "Programa de defensa para el Manejo Integrado de Plagas en Bananos y Plátanos". En este programa se orienta que aumentos de 200 unidades de Estado de evolución indican la necesidad de tratar con fungicidas en las próximas 72 horas de ser detectados (Pérez-Vicente et al. 2000; Centro Nacional de Sanidad Vegetal 2011).

Es importante destacar los niveles de EE que se observan en los meses de enero y febrero seguidos de un incremento en el mes de abril (Figura 1 y 2), lo que se diferencia del comportamiento epidemiológico que mostró la enfermedad por primera vez en Cuba en los años 1991 a 1993 y específicamente en el territorio que ocupa este trabajo según resultados de Pérez-Vicente et al. (2000), y que se puede considerar como línea base de referencia. Estos incrementos durante los tres primeros meses del año con elevados índices de estado de evolución, se reafirman si se considera la persistencia de condiciones meteorológicas en los diferentes escenarios (Figura 1 y 2) con niveles termofisiológicos superiores a $11000 \mathrm{u}$, considerados como propicios para el desarrollo de la enfermedad según criterios de PérezVicente et al. (2000).

La predicción epidemiológica de Sigatoka negra en estos años en Ciego de Ávila, avizora un incremento del costo de la protección fitosanitaria y la aplicación estricta del "Sistema de Manejo Integrado de Sigatoka negra", máxime que en la actualidad permanecen en explotación los mismos clones de bananos que se encontraban desde la aparición de la enfermedad y que además, se proyectan en los pla- nes futuros de desarrollo de este cultivo, susceptibles a la actividad fitopatógena de $M$. fijiensis.

Para comprender mejor la magnitud de los impactos esperados del cambio climático sobre el futuro desarrollo de Sigatoka negra en el cultivo del banano para este territorio, se hace necesario establecer diferencias con resultados de Pérez-Vicente et al.(2000) sobre el desarrollo epidemiológico de la enfermedad en el período comprendido de 1991 a 1993 en la propia Empresa de Cultivos Varios "La Cuba". Durante este periodo los índices del EE mostraron valores de alrededor de las 250 unidades, salvo momentos críticos como en diciembre de 1991 que alcanzó valores entre 1000 y 1250 u, todo lo que implicó que en 1991-1992 se realizaran 10 tratamientos, mientras en 1992-1993, los índices alcanzaron como máximo 750 unidades, por lo cual fue necesario recurrir a 13 tratamientos de fungicidas para poder interrumpir el desarrollo de la patología, aplicaciones bajo orientación de un sistema de señalización bioclimática en base a recurrencias de la enfermedad siempre que los índices superaron las 200 u del estado de evolución o cuando en las tres semanas posteriores a un tratamiento no se observó regresiones de la enfermedad (Pérez-Vicente et al. 2000).

Es oportuno destacar que las condiciones de temperatura y el régimen pluviométrico previstos en los escenarios justifican el comportamiento de la Sigatoka negra para los años 2020 y 2025 en Ciego de Ávila (Figura 1 y 2) y señalan condiciones para llegar a presentar un intenso desarrollo epidemiológico. Es significativo el aporte de estas variables climáticas en relación con los índices de humedad que requiere $M$. fijiensis para su germinación y crecimiento, que hacen posible completar su ciclo biológico con mayor rapidez y condicionar un buen desarrollo epidemiológico de Sigatoka negra. Aunque según Pérez-Vicente et al.(2000), a pesar de que 
se presenten índices de sumas térmicas inferiores a $11000 \mathrm{u}$, pueden no existir limitaciones en la velocidad de evolución en caso de incidir condiciones de alta humedad durante esos mismos períodos y así ocurrir rápidas recurrencias; agrega además que existe una fuerte relación entre los acumulados de 14 días de precipitación con los incrementos del estado de evolución 4 semanas posteriores (PérezVicente et al. 2000), elementos que se corroboran en las representaciones gráficas de los escenarios que se muestran en este trabajo.

Los resultados de esta investigación demuestran un incremento sistemático de la enfermedad en el trascurso de los meses del año, así como la permanencia de un incremento importante posterior a junio, fundamentalmente en los meses de octubre y noviembre. En comparación 2025 presenta índices de EE más elevados en el escenario A2, mientras los escenarios B2 presentan una disminución en referencia al 2020, no obstante, en ambos casos los niveles de la enfermedad son de interés y pueden ocasionar daños al cultivo, situación que se favorece de forma importante por los aumentos de temperatura debido al cambio climático, y que entra en correspondencia con lo expresado en los modelos del IPCC de no reducirse el reforzamiento radiativo mediante la mitigación.

Los escenarios bioclimáticos obtenidos imponen la necesidad de mantener durante todo el año, un ciclo constante de tratamientos con fungicidas en su mayoría con sustancias activas de acción sistémica y predominantemente en el período lluvioso, lo cual además de encarecer el costo de protección establece un alto riesgo para la aparición de razas resistentes. Esto, junto con las restantes consecuencias de pérdida de control de la enfermedad, hacen pensar que esta no será la solución más satisfactoria, por lo cual es de considerar en la planificación principios 67 de adaptación a una "Agricultura Climáticamente Inteligente" (según siglas en ingles CSA) que garanticen un eficiente control y manejo de esta enfermedad bajo formas sostenibles de producción, de conjunto con la mitigación y la adaptación al cambio climático y así contribuir a la seguridad alimentaria (FAO, 2013). Lo anterior se expresa en el requerimiento de empleo de tecnologías más limpias, en la utilización de sistemas de alerta, la señalización bioclimática de los tratamientos, el uso racional de fungicida, biopesticidas, bioestimulantes, y otras, además de proceder a la incorporación e intensificación práctica de medidas de manejo agroecológico como: la utilización de variedades y clones resistentes y certificados, el saneamiento permanente de hojas enfermas, evitar la colindancia con áreas comprometidas y con diferentes fechas de siembra, la correcta demolición total del cultivo al finalizar su ciclo productivo, implementar la lucha biológica mediante la búsqueda y aplicación de microorganismos que reduzcan las poblaciones del fitopatógeno, el aprovechamiento máximo de los recursos hídricos mediante la utilización de sistemas eficientes de riego, coberturas naturales, aplicación de materia orgánica que mejore las capacidad productiva, la fertilidad y la retención de la humedad en el suelo que permitan un buen ritmo de emisión foliar. También, el incremento de la biodiversidad a través del empleo de cercas vivas, policultivo, asociaciones y mosaicos de cultivo para evitar grandes extensiones y el monoclutivo, medidas que reducen el riesgo a la aparición fuertes epidemias pues coadyuvan a la disminución de las fuentes de inóculos, la restricción de la presión y así evitar el acortamiento del período de incubación del hongo, con ello el desarrollo de la enfermedad, sus índices de infección, los daños a la plantación y ganar en resilien- 
cia en el agroecosistema ante el impacto del cambio climático.

Es posible concluir a partir de la interpretación de los escenarios bioclimáticos para los años 2020 y 2025 bajo condiciones de cambio climático que las proyecciones futuras expresadas mediante criterios termofisiológicos (SV) en función de la temperatura máxima y mínima, así como por la determinación del EE en función del comportamiento pluviométrico, señalan una intensificación del desarrollo epidemiológico de Sigatoka negra en bananos, que ponen en riesgo la producción de esta fruta con repercusión para la seguridad alimentaria en Ciego de Ávila y en Cuba.

Ante el futuro incremento epidemiológico de la Sigatoka negra en la áreas productivas en Ciego de Ávila, se prevé la probabilidad de: la elevación del costo de la protección fitosanitaria y de producción, incidencia de pérdidas de rendimiento en sistemas agrícolas convencionales y afectaciones de extremo impacto en los sistemas orgánicos que contengan (variedades susceptibles) y que incumplan con el manejo agroecológíco.

Se recomienda la aplicación de una "Agricultura climáticamente Inteligente" que contenga medidas, tecnologías y acciones dirigidas a reducir las emisiones de gases de efecto de invernadero que permita la adaptación al cambio climático y la sostenibilidad productiva, con énfasis en el rediseño de los agro ecosistemas, desarrollar una transición de sistemas convencionales de producción de banano a sistemas biodiversos y resilientes con mayor sostenibilidad, donde el control de la enfermedad se ejerza mediante la aplicación de medidas de manejo, que contenga agroecológicas, y ser incluidas dentro de las políticas de gestión para el enfrentamiento al cambio climático y en los programas de adaptación como elementos claves de la planificación y establecimiento de los sistemas de explotación banane- ros. De esta forma se logrará alcanzar una mayor resiliencia y condicionantes que permitan luchar contra esta patología en bananos, que coadyuven a la reducción y la presión de las fuentes de inóculos, evitar el acortamiento del período de incubación del hongo para impedir el desarrollo de la enfermedad, la reducción de sus niveles de infección, los daños a la plantación y preservar los índices productivos y poder mantener un producto al alcance de todos los cubanos, con contribución a la seguridad y soberanía alimentaria.

\section{Conflictos de intereses}

Los autores han cumplido las normas éticas de publicación, y no generan conflicto de interés en la presente investigación.

\section{Agradecimientos}

Se agradece al Centro de Meteorología Agrícola y al Centro de Física de la Atmósfera del Instituto de Meteorología la facilitación y acceso a las salidas del Modelo Climático Regional PRECIS-CARIBE. En especial a los investigadores Dr. Oscar Solano Ojeada y MSc. Ranses Vázquez por su cooperación.

\section{Literatura citada}

Altieri MA, Nicholls CI. Los impactos del cambio climático sobre las comunidades campesinas y de agricultores tradicionales y sus respuestas adaptativas. Agroecología. 2008; 3:7-24.

Carter TR, Parry ML, Harasawa H, Nishioka S, Christ R, Epstein P, et al. Technical Guidelines for Assessing Climate Change Impacts and Adaptations. En: Houghton T, Meira Filho LG, Bruce J, Lee H, Callander BA, Haites E, et al, 
editores. Climate change 1994: radiative forcing of climate change and an avaluation of the IPCC IS92 emission scenarios [Internet]. Londres: University College; 1994. p. 829-33. [citado 10 Abr 2016]. Disponible en: http://www.ipcc-wg2.gov/publications/SAR/ SAR_Chapter\%2026.pdf.

Centro Nacional de Sanidad Vegetal, Subdirección de Protección de Plantas, Dpto. Programas de Defensa. Programa de defensa para el manejo integrado de plagas en bananos y plátanos [Internet]. La Habana: Ministerio de la Agricultura, 2011 [citado 10 Abr 2016]. Disponible en: http://www.actaf.co.cu/index.php?option=com _mtree\&task=att_download\&link_id=294\&cf_ $\mathrm{id}=24$.

Cline WR. Global warming and agriculture. impact estimates by country, Washington: Centre for Global Development; 2007.

De Schutter O. Promotion and protection of all human rights, civil, political, economic, social and cultural rights, including the right to development. Report submitted by the Special Rapporteur of UNESCO on the right to food, Olivier De Schutter [Internet]. New York: United Nations General Assembly; 20 Dic 2010. Sixteenth session. Agenda item 3 [citado 10 Abr 2016]. Disponible en: http://www. srfood.org/images/stories/pdf/officialreports/20 110308 a-hrc-16-49 agroecology en.pdf.

Dirección de Cultivos Varios. Informe del cultivo del plátano y banano. Ciego de Ávila: Delegación Territorial de la Agricultura en Ciego de Ávila, Subdelegación de Cultivos Varios; 2011.

FAOSTAT: Organización de las Naciones Unidas para la Alimentación y la Agricultura. Dirección de Estadísticas [Internet]. Roma: FAO c2015-. [citado 24 Oct 2016]. FAOSTAT Descargar Datos; [aprox 2 p.]. Disponible en: http://faostat3.fao.org/download/Q/QC/S.

Food and Agriculture Organization of the United Nations. Climate-Smart Agriculture Sourcebook [Internet]. Roma: FAO; 2013. [citado 24 Oct 2016]. Disponible en: http://www.fao.org /docrep/018/i3325e/i3325e.pdf.

Fouré E. Les Cercosporiose du bananier et leurs traitement. Comportment des varietés. Etude de la sensibilité varietale des bananiers et plantains a Mycosphaerella fijiensis Morelet au Gabon (maldie de raies noires). I. Incubation et evolution de la maladie. II etude de quelques parametres. Fruits. 1982; 37:749-54.

Intergovernmental Panel on Climate Change. Cambio climático 2007: Informe de síntesis. Contribución de los Grupos de trabajo I, II y III al Cuarto Informe de evaluación del Grupo Intergubernamental de Expertos sobre el Cambio Climático. Ginebra: IPCC; 2007.

Lecha Estela LB, Paz Castro LR, Lapinel Pedroso BP. El clima de Cuba. La Habana: Editorial Academia; 1994.

Nakičenovič N, Alcamo J, Davis G, de Vries B, Fenhann J, Gaffin S, et al. Special Report on Emissions Scenarios. A Special Report of Working Group III of the Intergovernmental Panel on Climate Change. Cambridge: Cambridge University Press, 2000.

Pérez-Vicente L, Mauri Mollera F, Hernández Mancilla A, Abreu Antúnez E, Porras-González A. Epidemiología de la Sigatoka negra (Mycosphaerella fijiensis Morelet) en Cuba. I. Pronóstico bioclimático de los tratamientos de fungicidas en Bananos (Musa acuminata AAA). Rev Mex Fitopatol. 2000; 18(1): 15-26. 
Porras-González A, Pérez-Vicente L. Efecto de la temperatura sobre el crecimiento de los tubos germinativos de las ascosporas de Mycosphaerella spp., causantes de Sigatoka en banano. Cálculo de las sumas de velocidades de desarrollo para el pronóstico de los tratamientos a partir de la temperatura mínima y máxima diarias en Cuba. Infomusa. 1997; 6(2): 27-32.

Romero, R. Control de Sigatoka negra en bananos y plátanos en Costa Rica. Simposium BASF sobre control de Sigatoka negra en banano. Jul 1993; Villa Hermosa, México. Villa Hermosa; 1993.

Rosenzweig C, Casassa G, Karoly DJ, Imeson A, Menzel A, Rawlins S, et al. Assessment of observed changes and responses in natural and managed systems. En Parry ML, Canziani OF, Palutikof JP, van der Linden PJ, Hanson CE, editores. Climate Change 2007: Impacts, Adaptation and Vulnerability. Contribution of Working Group II to the Fourth Assessment Report of the Intergovernmental Panel on Climate Change [Internet]. Cambridge: Cambridge University Press; 2007 p. 79-131. [citado 10 Abr 2016]. Disponible en: https://www.ipcc. ch/pdf/assessment-report/ar4/wg2/ar4-wg2chapter1.pdf.
Stover RH, Simmonds NW. Bananas. 3ra ed. Singapur: Lonngman Scientific \& Tecnical; 1987.

Stover RH. Sigatoka leaf spots of bananas and plantains. Plant Dis. 1980; 64: 750-5.

Taylor MA, Centella A, Charlery J, Bezanilla A, Campbell J, Borrajero I, et al. The precis Caribbean story lessons and legacies. B Am Meteorol Soc. 2013; 94(7): 10651073.

Vidal A. Sigatoka negra en Cuba. En nuevos focos de plagas y enfermedades. Boletín Fitosanitario de la FAO. 1992; 40: 1-2.

Warrington J. Climate change and horticulture. Chron Horticult. 2008; 48(1):1-2. 